\title{
Das Meer: Filmische Wahrnehmung und das Unermessliche
}

\author{
Heller, Franziska
}

Posted at the Zurich Open Repository and Archive, University of Zurich ZORA URL: https://doi.org/10.5167/uzh-44997

Book Section

Originally published at:

Heller, Franziska (2010). Das Meer: Filmische Wahrnehmung und das Unermessliche. In: Mauer, R. Das Meer im Film. Grenze, Spiegel, Übergang. München: Edition text + kritik, 45-60. 


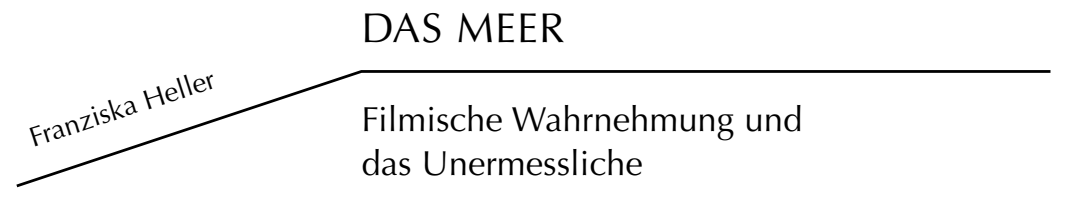

Herrlich ist es, in einer unendlichen Einsamkeit am Meeresufer, unter trübem Himmel, auf eine unbegrenzte Wasserwüste, hinauszuschauen. Dazu gehört gleichwohl, (...) daß man alles zum Leben vermisst, und die Stimme des Lebens dennoch im Rauschen der Flut, im Wehen der Luft, im Ziehen der Wolken, dem einsamen Geschrei der Vögel, vernimmt. Dazu gehört ein Anspruch, den das Herz macht, und ein Abbruch, (...), den einem die Natur tut. Dies aber ist vor dem Bilde unmöglich, und das, was ich in dem Bild selbst finden sollte, fand ich erst zwischen mir und dem Bilde, nämlich einen Anspruch, den mein Herz an das Bild machte, und einem Abbruch, den mir das Bild tat (...). ${ }^{1}$

Heinrich von Kleist

\section{Bilder und das Meer: Anspruch und Abbruch}

Im Angesicht einer Seelandschaft verdichtet sich ein Geflecht aus widersprüchlichen Eindrücken zu einer Empfindung. Anschaulich und einfühlsam beschreibt Heinrich von Kleist, welche Erfahrung er bei der Betrachtung von Caspar David Friedrichs Gemälde Der Mönch am Meer (1808-10) macht. Man vermisst das Leben und erlebt es doch akustisch im Rauschen der Flut. Die widersprüchlichen Empfindungen entstehen aus der gleichzeitigen Erfahrung von An- und Abwesenheit. Dies übersetzt sich gar in das Wahrnehmungsdispositiv des Bildbetrachters. Kleist spricht von einem Anspruch, den sein Herz an das Bild heranträgt. Das Bild aber in seiner ästhetischen Struktur und darstellenden Dimension kann diesen Anspruch nicht gänzlich erfüllen. So ist entscheidend, dass die Empfindung gegenüber Friedrichs Meer als eine Erfahrung des Defizits entsteht. Diese Erfahrung entwickelt sich zwischen dem Betrachter und der ästhetischen Struktur als eine ständige sinnliche Bewegung zwischen Anspruch und Abbruch. 
Schon bei Kleist findet sich also in der Widersprüchlichkeit der Eindrücke ein Bewegungselement, das grundlegend für die ästhetische Wahrnehmung des Meeres ist. Dieses nun an filmischen Bildern zu untersuchen, erscheint aufgrund ihrer besonderen Bewegungsqualität reizvoll. Es stellt sich die Frage, wie das Wahrnehmungsdispositiv des Meeres als nicht fassbares Bewegungsuniversum in filmischen Bildern funktioniert. Die Konsequenzen führen zu rezeptionsästhetischen Überlegungen: Es geht um den Abbruch, mit dem das (filmische) Bild den Betrachter konfrontiert, und zugleich um den Anspruch, der dadurch filmisch und erzählerisch erfüllt wird.

\section{Wandelnde Wahrnehmung des Meeres in der Kultur- und Geistesgeschichte}

Das Meer, wie auch Wasser generell, unterliegt auf der ikonografischen Ebene einer breiten Heterogenität. ${ }^{2}$ Die Heterogenität, manchmal gar Polarität der Eindrücke, drückt sich vor allem in der Variabilität der symbolischen wie sinnlichen Konnotationen aus. Dabei lässt sich beobachten, dass insbesondere das Meer eng mit körperlichen und räumlichen Erfahrungsweisen verbunden ist, wobei immer wieder die Konfrontation mit dem Unermesslichen thematisiert wird: räumlich wie zeitlich.

So können die Erfahrungen des Meeres positiv, zugleich aber auch zutiefst negativ sein. Beide Bewertungen setzen an Grundkoordinaten unserer Existenz an. Das Meer vermag ein mythisches Bild der Apokalypse zu verkörpern, das auf Urängsten und Urerfahrungen der Menschheit gründet. ${ }^{3}$ Um diese Urerfahrungen ranken sich unterschiedlichste Diskurskreise vor allem in der christlichen Ikonologie. Das Meer bietet etwa das räumliche Dispositiv für das Konzept vom „Schiff der Kirche“ an, welches Sicherheit gewähren soll. Kraft und Erlösung vom Untergang sowie Auferstehung werden im Akt der Taufe angeboten als Ausdruck der Erneuerungskraft des Lebens. ${ }^{4}$ Das Meer als chaotischer Bewegungsraum wie als unerschöpfliche Quelle von Lebenskraft - beide Vorstellungen inkorporieren Empfindungen der Unfassbarkeit (von deren Immensität der Bewegung jeweils die Kirche als Stabilisator profitiert).

Die Wandelbarkeit der Wahrnehmung des Meeresmotivs ist eng mit geistesgeschichtlichen Konstruktionen des Subjekts und seinem Verhältnis zur Welt verbunden. So markiert Bernhard Blume etwa den tiefsten Einschnitt für den ästhetischen Ausdruck von Wasserbewegungen, ähnlich wie es Hartmut Böhme für die Kulturgeschichte tut, zu Beginn des 18. Jahrhunderts. ${ }^{5}$ Hier vollzieht sich eine Wende vom Jenseits zum Diesseits. Die 
allegorische Gleichsetzung von Schifffahrt und Lebensreise, von Meer und Dasein tritt zurück. Stattdessen erscheinen Flüsse und Seen; man wendet sich zum Bekannten, Begrenzten, zum Endlichen, bei dem das Ufer in Sicht bleibt. ${ }^{6}$ Die endlose Fläche des Meeres ist dagegen wiederkehrendes Element des Unüberschaubaren, da nicht mit dem Auge vollständig erfassbar.

Bleibt man bei der Problematik der Über-Schaubarkeit angesichts des Meeresraumes, so muss man eine besondere Eigenschaft hervorheben, die im Kontext des Filmischen zentral wird: Das Meer manifestiert sich ästhetisch fast immer in einem relationalen Verhältnis zu etwas Festem oder Stabilem. So sind insbesondere "Meer" und „Insel“ (bzw. auch „Küste“) tatsächlich gesehene und erfahrene Gegensätze, wobei die Grenzenlosigkeit, Formlosigkeit und die Bewegung des Meeres sich in dem Verhältnis zur Begrenzung, Einschließung und Ruhe der Insel artikulieren. Diese (ästhetischen) Eindrücke funktionieren vor allem zusammen. Sie müssen in einem einzigen Bild ${ }^{7}$ zusammen gesehen werden und generieren so eine in sich gegensätzliche Einheit des Eindrucks. Es entsteht eine in sich dynamische, da auf differenzieller Bewegung basierende, bildhafte Polarität. ${ }^{8}$ Ähnlich bipolar funktionieren die Bilder von „Meer“ und „Schiff“ mit einem weiten metaphorischen Potenzial: Der Mensch kann (körperlich) in diesen Bildern als Seefahrer, schwimmend, fahrend, scheiternd, ertrinkend, mit dem Elementaren konfrontiert werden. ${ }^{9}$

\section{Filmische Wahrnehmungsstrukturen angesichts des Meeres}

Das Meer als weiße Leinwand der Wahrnehmung: Entzug von Innen(ansichten)

Im Kontext von Michelangelo Antonionis L'Avventura (Die mit der Liebe spielen, F/I 1960) wird auf eine Sequenz eingegangen, die den Vorlauf zu dem berühmten Schlussbild - Claudia und Sandro wortlos angesichts der Meeresfläche - darstellt. In dem Beispiel vollzieht sich eine paradoxe Polarisierung durch die Auflösung der vermeintlichen Innenräume. Diese geschieht mithilfe einer Öffnung der filmischen Bilder über die Bewegung des Meeres - eine ständige Öffnung in das unbestimmte Außen des Meeres, das buchstäblich als visueller weißer Fleck, ja fast als weiße Leinwand für die Vorstellung des Zuschauers offengelassen wird. Die Weite des Meeres erfasst dabei phänomenologisch die geometrischen Prinzipien der hallenartigen Räume und Flure. Dieses Widerspiel der Eindrücke vermit- 
telt die Isolation - etymologisch auf "Isola“ verweisend - der Figuren als raum-zeitliche Erfahrung. Auf diese Weise erzählt Antonioni sein Sujet der entleerten Existenz.

Der Film zeigt die Ereignisse in der illustren Vergnügungsgesellschaft um die Freundinnen Claudia (Monica Vitti) und Anna (Lea Massari) sowie deren Verlobten Sandro (Gabriele Ferzetti). Die drei brechen mit einer gut betuchten Gesellschaft zu einer Jacht-Tour auf. Auf einer Insel verschwindet Anna spurlos. Die Suche nach Anna rückt zunehmend in den Hintergrund und bildet die Folie für die Orte, an denen sich Sandro und Claudia aufeinander zu bewegen und wieder voneinander entfernen. Schließlich fahren sie zu einem Hotel, wo ein Fest stattfindet. Während Sandro noch auf das Fest geht, wartet Claudia in ihrem Zimmer. Dabei sieht man sie meist von Türrahmen kadriert, wodurch ein verdichteter Raumeindruck entsteht. Dann tritt Claudia auf die Terrasse. Man schaut über ihre Schulter in die Bildtiefe und auf das weite Meer hinaus. Claudia verdoppelt den Zuschauerblick, indem sie in Rückenansicht auf die zur Kontemplation einladende Meeresweite starrt. ${ }^{10}$ Dem Betrachter wird dadurch angeboten, ihren Blick, den er nicht sieht, imaginär zu ergänzen und somit ihren unfokussierten Blickmodus in die Weite nachzuvollziehen. Daraufhin geht Claudias schattenhafte Kontur nach rechts aus dem Bild, ganz nah an der Kamera vorbei.

Antagonistisch zu dieser konkreten visuellen Nähe zu Claudia und der imaginären Nähe, die durch die Identifikation mit ihrer Blickrichtung

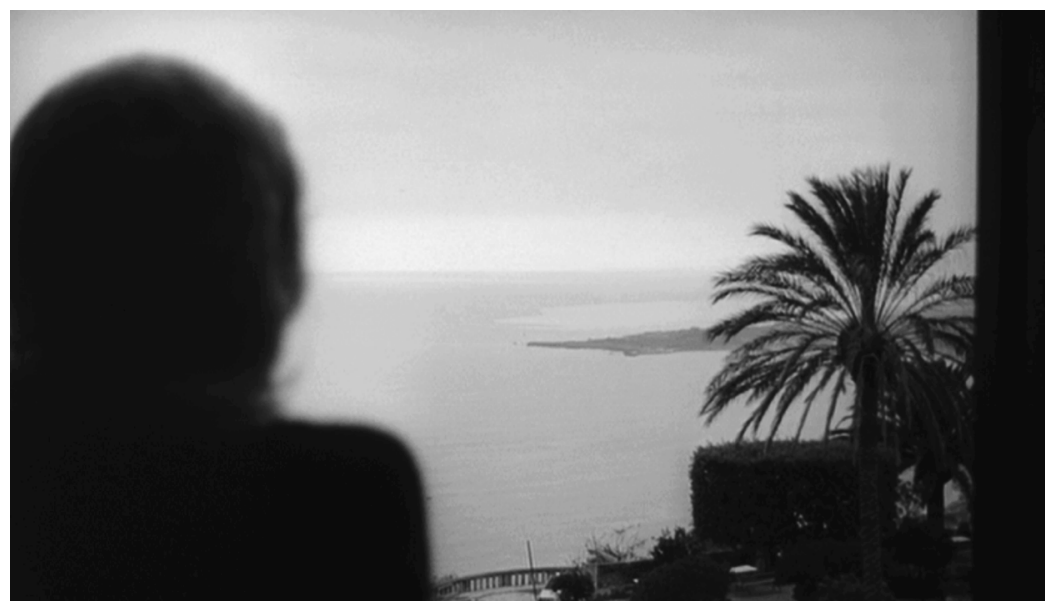

L'Avventura (Die mit der Liebe spielen, F/l 1960), R: Michelangelo Antonioni 
entsteht, verhält sich die Einstellung nach dem Umschnitt: Denn nun ist Claudia das Objekt des suchenden Zuschauerblickes. Sie befindet sich nun weit hinten in der hohen zentralperspektivischen Flucht eines Flures und ist kaum zu erkennen. Ihr Vorwärtslaufen verliert sich in der Ferne, wodurch die Bewegung auch in der Raumerfahrung des Zuschauers verlorengeht. Die Montage gegensätzlicher räumlicher Eindrücke von Nähe und Ferne, der Weite des Meeres und der Länge des Flures ermöglicht die intensive Erfahrung der Isolation dieser Figuren.

Als Claudia einen anderen Raum betritt, beginnt man das Rauschen des Meeres zu hören. Im nächsten Zimmer scheint das Meeresrauschen noch aufdringlicher zu werden. Je weiter Claudia im Raum entlanggeht, desto mehr sieht man von der großen Glasfront, die auf ein undefiniertes weißes Außen hinausweist. Eine Glastür zur Terrasse steht offen. In einem anderen Raum schreitet Claudia an einer langen Tafel entlang. Dahinter sind große Spiegel. Diese erinnern an eine Trompe-l'oeil-Konfiguration, die Fenster imitiert: Sie richten den Blick nicht in ein verortendes Außen, sondern werfen das Innere auf sich selbst und den (innerfilmischen) Betrachter zurück. Umso unbestimmter wird das Außen durch das Meeresrauschen. Claudia schaut durch einen Torbogen. Sandro liegt auf einer Couch mit einer Prostituierten. Nach einem Moment bemerkt er Claudia. Währenddessen ist die ganze Zeit das Rauschen des Meeres zu hören. Musik gibt es keine. Claudia rennt den langen Tisch entlang aus dem Bild.

Die beschriebene Sequenz beginnt mit der Fokussierung auf Claudia als Blicksubjekt. Ihr Blick führt den Zuschauer auf einer gedoppelten Ebene (eigener Blick und Blick der Figur) in die Weite des Meeres, wobei Claudia dem Zuschauer als menschlicher Anhaltspunkt im Raum Nähe und Fixierung vermittelt. Sowohl diese Nähe wie auch der Eindruck der Weite des Meeres bilden die Folie für die folgende Konterkarierung dieser Eindrücke. Der Blick wird zentralperspektivisch verengt und die Nähe zu Claudia geht so verloren. Auf diese Weise wird für den Zuschauer ein Verlust erfahrbar. Dies ist das generelle Prinzip der interagierenden dialektischen Raumeindrücke: Sie vermitteln im Zuschauerhorizont das Gefühl einer begrifflich nicht fassbaren Verlorenheit von Sinn und festem Raumgefühl.

Dabei spielt das Motiv der „Insel“ ideell eine maßgebliche Rolle. Die Insel steht als fester Punkt in einer undefinierbaren, dynamischen Weite. Claudia ist der sich in ständiger Bewegung befindende Bezugspunkt im Raum, wird aber dem Zuschauerblick immer wieder entzogen. Dieses „Entziehen" geschieht zugleich auf einem Schauplatz, der durch seine Mauern und die Spuren wohnlicher Elemente auf einen Innenraum verweist. Fixierende Kategorien werden offensichtlich im Bild immer nur noch ,an- 
zitiert“. Allerdings sehen sich die Anhaltspunkte ständig sinnlich konterkariert. Durch das Meeresrauschen wird der Wahrnehmungsraum des Zuschauers ins Unbestimmte geöffnet. Visuell übersetzt sich diese Unbestimmbarkeit des Raumes in den weißen Flächen der Fenster, die nach außen deuten, aber die Fassbarkeit des Außen negieren. Es ist das erfahrene Paradox einer Fixierung, die in einer (auditiven) Bewegung durch eine unbestimmbare Weite fortdauernd destabilisiert wird. Das Meer als relationales Wahrnehmungselement ermöglicht die sinnliche Entzugsbewegung. Ihr unterliegen die Wahrnehmung von Innenräumen, aber auch die (Innen-)Ansichten auf die Figuren.

\section{Kontrollverlust der Wahrnehmung:}

Der leblose Körper im Meer

Eine solche Verlusterfahrung von Stabilität angesichts des Meeres kann sich auch auf das (filmische) Subjekt richten, insbesondere wenn der menschliche Körper willenlos und bewegungsunfähig dem Meer ausgeliefert ist. Die in L'Avventura noch angedeutete räumliche Stabilität, die den orientierenden Ausgangspunkt für den Verlust darstellt, wird nun durch den menschlichen Körper ersetzt. Auf die Weise wird der scheinbare Fixpunkt im Bild beweglicher, aber zugleich noch unverlässlicher als vermeintliche Innenräume.

In The Bourne Identity (Die Bourne Identität, USA/BRD/CS 2002) spielt der filmische Topos des menschlichen Körpers, der im Meer treibt, eine nahezu leitmotivische Rolle: Das Universum von Jason Bourne (Matt Damon) wird schon in der Exposition paradigmatisch auf der raum-zeitlichen Erscheinungsweise als diffuses Bewegungschaos eingeführt. Der Film spielt nachhaltig mit dem Erleben zwischen Kontrolle und Verlust der Kontrolle über das Wahrnehmbare. Dies hat seine inhaltliche Entsprechung: Die Suche von Jason Bourne nach seiner Identität ist eng mit der Les- und Erkennbarkeit seiner Umwelt verwoben.

So beginnt der Film in Dunkelheit: Ein Blitz eröffnet den momenthaften Blick in den diffusen Unterwasserraum eines sturmgepeitschten Meeres. Ungewöhnlich tief mit der Kamera unter der Wasseroberfläche hängend, erahnt man am linken Bildrand, zwischen dem wilden Wellentreiben, einen verschwommenen Körper. Man hat kaum Zeit, sich zu orientieren. Die Wellen an der luziden Oberfläche treiben mit immenser Kraft durch das Bild. Untermalt von Donner hält sich die Einstellung nur für einen Moment, um dann von einer Einstellung über der Meeresfläche abgelöst zu werden. Die schweren, aber zugleich treibenden Bewegungen der Wel- 


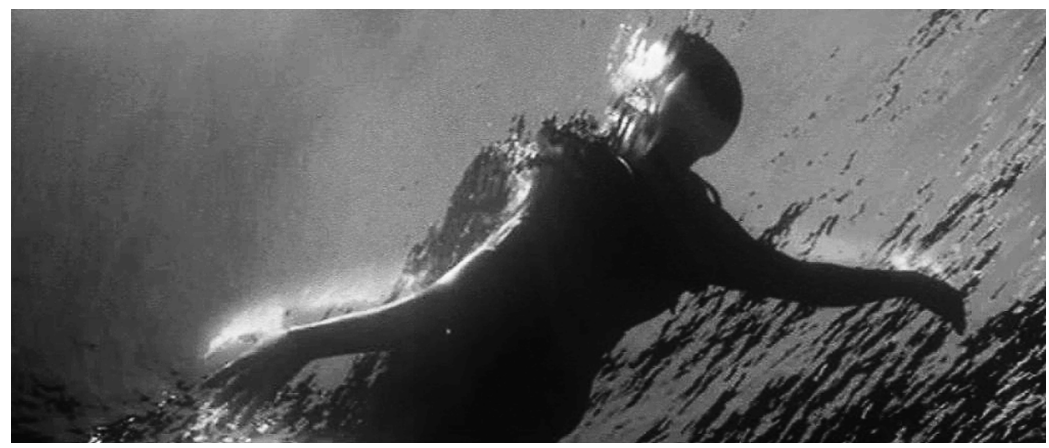

The Bourne Identity (Die Bourne Identität, USA/BRD/CS 2002), R: Doug Liman

len, nun in der Aufsicht, schließen an das vorherige Wogen der Wassermassen an. Die Nacht ist tiefblau, Regen löst die Sicht auf. Ein Blitz erhellt kurz die peitschende Szenerie, um sie dann bei seinem Verlöschen in noch größere Diffusität zu stürzen.

Gegen diese wilde Umgebung werden die Innenaufnahmen aus einem Schiff gesetzt, die die raue Schiffsbesatzung beim Kartenspiel in satten braun-feuchten Farbtönen zeigen. Der Blick ist beengt durch Kadrierungen und Bildausschnitte: ein krasser Gegensatz zu der sturmgepeitschtenWeite draußen. Dagegen wird wieder in eine tief gesetzte Untersicht aus dem Meer geschnitten; etwas näher als vorher. Unterschiedliche Wellenformationen überlagern sich an der Oberfläche. Der Eindruck eines kraftvollen, undurchdringlichen Treibens entsteht. Als ein Mann der Schiffsbesatzung sich hinaus in den Regen quält, spiegelt sich in seinen Brillengläsern das punktuelle Blinken des Senders am Körper. Dabei setzt im Hintergrund das musikalische Leitmotiv von Jason Bourne ein. Für einen Moment ist die Kamera ganz dicht, leicht schräg unter der Wasseroberfläche. Sie fährt an dem Körper entlang: Er liegt auf dem Rücken, beide Arme weit von sich gestreckt. Bevor die Kamera ihn erreicht, geht das Bild in einem Black auf. In fahl-blauen Farbtönen materialisiert sich der Titel des Films.

Bis hierhin sind knapp anderthalb Minuten Laufzeit vergangen, doch zentrale Topoi und Strukturelemente des Films wurden etabliert: Die Exposition ist gekennzeichnet durch einen immensen Bewegungsdrang. Die Wellenformationen und das dauerpräsente harte Einprasseln des Regens vermitteln eine heftige Unruhe, eine unkontrollierbare Bewegung der Welt. Der Körper Jason Bournes ist der vollen Kraft der Naturgewalt ausgesetzt: im Wasser und vom Himmel; auf der Horizontalen wie auf der Vertikalen. Zum einen findet sich der Zuschauer in dem materiell schwer fass- 
baren Unterwasserraum wieder, in den man mit der Kamera tief eintaucht. Man sieht sich mit der Wasseroberfläche konfrontiert, die nur als immense Bewegungsform wahrgenommen wird. Die Entfernung zu ihr ermöglicht die breite Ansicht auf die raumgreifende Omnipräsenz des unkontrollierbaren Wogens.

Das Innen des Schiffes ist zwar mit warmen Tönen versehen, vermittelt aber zusammen mit den fragmentarisierenden Bildausschnitten eine ständige Unruhe. Die letzte Fahrt, knapp unter der Wasseroberfläche, verdichtet noch einmal das Gefühl von jagender, äußerer Bewegung. Es erscheint wie ein Sinnbild von Jason Bournes ohnmächtiger Situation. Dabei kann der Zuschauer sein Gesicht, Ausdruck seiner Identität als Filmheld, nur hinter der fluiden Fläche erahnen. Ähnlich wird es Bourne inhaltlich ergehen. Er ist einem ahnenden Zwischenzustand zwischen Erinnerung, Vergessen und aktueller, nicht zu dechiffrierender Situation ausgeliefert.

Dieses Bewegungschaos des Meeres wird im Verhältnis zu Bournes Körper inszeniert. Der menschliche Körper selbst, Ausdruck von ikonischer Identität filmischer Protagonisten, hat keine eigene selbstbestimmte, intentional gerichtete Bewegung: Er ist kaum sichtbar, von einem anderen Bewegungsmedium umfangen. Dies übersetzt sich für den Filmzuschauer in die konkreten Prinzipien der Sichtbarmachung, die nur für blitzhafte Augenblicke kurze „Ein-Sichten“ ermöglichen, diese damit versprechen, um sie sofort wieder zu entziehen. Es ist ein Kontrollverlust über die veranschaulichende Zeige-Dimension filmischer Bilder. Das Meer in dieser Eröffnungssequenz ist somit das phänomenologische Medium für die Vermittlung eines filmischen Universums, das seine Unkontrollierbarkeit und Unfassbarkeit insbesondere für den Filmzuschauer als Bewegungsdrang ausstellen will.

Die Tiefe des Meeres und die filmische Dauer

Die Erfahrung eines filmischen Entzugs kann auch in eine besondere zeitliche Dimension transponiert werden: In John Hustons Moby Dick (USA 1956) wird über die bildlichen Sichtbarkeitsstrukturen im Bewegungschaos des Meeres eine filmische Dauer vermittelt. Auf diese Weise wird eine unglaubliche Zerstörungskraft erzählt. Dies ist umso bemerkenswerter, da es über die Vorstellung der Kraft und Tiefe des Meeres geschieht, die in eine kreisende Bewegung übersetzt wird.

Die zitierte Sequenz setzt da ein, wo Ahab (Gregory Peck) bereits umgekommen ist. Überraschenderweise übernimmt nun Starbuck (Leo Genn) das Kommando. ${ }^{11}$ Während die kleinen, wankenden Fangboote anfangen 
zu pullen, schweben die Möwen bereits bedrohlich über ihnen. Plötzlich taucht Moby Dick unter einer riesigen Gischtfontäne auf. Das Chaos bricht aus - ein Boot wird auf seinem Rücken zum Kentern gebracht, spritzende Gischt beherrscht von nun an die Bilder und lässt jegliche Wahrnehmung diffus werden. Immer wieder sieht man nur Ausschnitte des Ungetüms, das durch das aufgewühlte Wasser gleitet. Das Meer, das den Großteil der Bildfläche bestimmt, wird zu einem heterogenen Raum aus sich bedrohlich auftürmenden Wellenbergen. Die Männer schreien in Panik. Zwischen der auf die Kamera einfallenden Gischt erkennt man momenthaft ein sich öffnendes Maul, das auf die Kamera zukommt. Zähne blitzen auf. Gischt ist überall. Moby Dick taucht unter die Pequod, nur um dann wieder unter einem riesigen Schwall aus dem Wasser aufzutauchen. In einer Totalen sieht man nun, wie das herrenlose Schiff anfängt, sich um sich selbst zu drehen. In Nahaufnahmen gleiten Fragmente von Moby Dicks Körper hin und her durch die Bilder. Durch die Montage der unterschiedlichen Bewegungsrichtungen wirkt das Zerstörungswerk allumfassend und allgegenwärtig. Das aufgewirbelte Wasser überspült hierbei die Kamera, die nun auf Meeresspiegelniveau abgesunken ist. Damit gibt die Kamera „überschauende" Perspektiven zunehmend auf. Die Spitze des Schiffes ist schon unter Wasser - sie hat sich unablässig in das Meer hineingedreht.

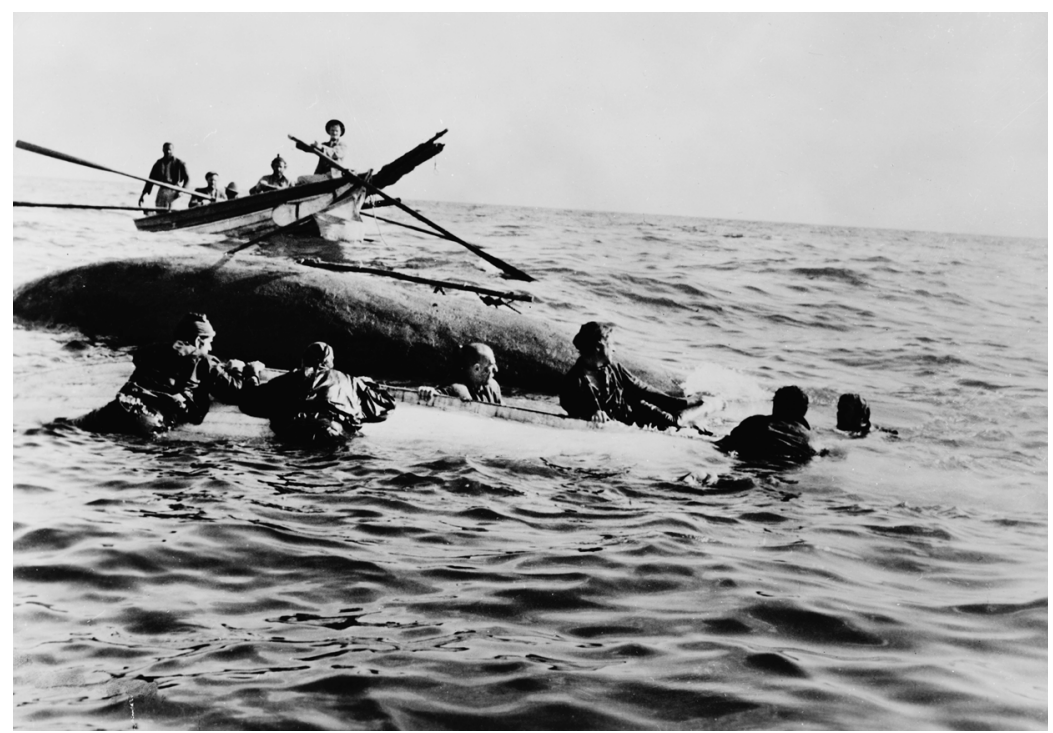

Moby Dick (USA 1956), R: John Huston 
Die Möwen kreisen kreischend darüber, nun näher. Nur noch das Heck des Schiffes ragt aus dem Wasser, aber es dreht sich erbarmungslos weiter. Unter einem triumphierenden Aufbäumen Moby Dicks und einer weiteren hohen Gischtfontäne wird das Schiff vom Meer verschluckt. Die Kamera wankt wogend und verunsichert. Was dann bleibt, sind die um sich drehenden Wirbel - fluide Andeutung der unkontrollierbaren Kraft der Strömung und Zerstörung, die alles unwiederbringlich in die Tiefe gezogen hat. Dieser Moment wirkt so intensiv, da sich die Kamera in Nahaufnahme auf die Strömung und ihre unvorhersehbaren, nicht kontrollierbaren Bewegungen konzentriert. Dann ist alles verschwunden, nur die letzten Bewegungen des Wassers scheinen ein wogendes Echo der vorherigen Apokalypse zu sein. Im Gischtnebel erscheint plötzlich als einziger Überlebender Ismael (Richard Basehart).

Das Meer als ewig bewegter Raum bildet das zentrale Wahrnehmungsdispositiv des Films, das sowohl inhaltlich thematisiert als auch strukturell von Huston filmisch profiliert wird. Dabei ist für seine Inszenierung wichtig, dass das Meer der Wahrnehmung keine feste konkrete Orientierung bietet. ${ }^{12}$ Alles ist in ständiger Bewegung, verortende Kategorien wie Nähe und Ferne lassen sich nur abstrakt und relational zur eigenen Position bestimmen und konstruieren - nicht durch gegebene, stabile Objekte. So ist das Schiff verortendes, aber zugleich bewegtes Zentrum der Obsessionen, der Glaubenskonfrontationen und Wahrnehmungstäuschungen gewesen. Es ist der Mikrokosmos, in dem jegliche raum-zeitliche Verortung und Zielsetzung zusammenlaufen. Angesichts der beängstigenden Unermesslichkeit des Meeres resultiert daraus für die Besatzung ein Zurückgeworfensein auf das Schiff. Auf der Ebene des Zuschauers spielt dies alles am Ende zusammen: Der sich drehende Strudel generiert sinnlich die Konfrontation mit der Unermesslichkeit. Als Resultat des Kampfes mit einer unfassbaren Naturgewalt (dem Meer wie dem Wal) wird filmisch der kreisende und dadurch dauernde Untergang in die Tiefe des Meeres vermittelt. In dieser Erfahrung der Dauer wird dem Zuschauer mit dem Schiff auch der Anhaltspunkt der Wahrnehmung genommen. Zudem provoziert der Film ein Gefühl der Unentrinnbarkeit: Die Kamera wird ständig überspült, die Bildfläche von dem wogenden Meer bestimmt und selbst der Himmel von den zuckenden Bewegungen der Möwen eingenommen. In der Dauer des Untergangs und der Erfahrung des unentrinnbaren Chaos wird schmerzhaft, Stück für Stück, der Verlust von etwas sinnlich Unermesslichem erfahrbar. Was bleibt ist ein einziger, nicht fassbarer Bewegungsraum, der über das Bild hinausgeht - gerade weil es bei dem Zuschauer in dem erfahrenen Verlust nachhallt. 
Das Meer und filmisches Begehren

Lucía yel sexo (Lucía und der Sex, F/E 2001) wird hier abschließend besprochen, da das filmische Universum, das Regisseur Julio Medem generiert, nach den Bewegungsprinzipien eines wogenden Meeresraums funktioniert. Dies wird direkt in die Wahrnehmungsbilder überführt, noch radikaler als in The Bourne Identity - und mit dem pikanten Zusatz, dass Themen der Sehnsucht und des Begehrens erzählt werden. Der formalästhetische Clou liegt darin, für den Zuschauer das Objekt der Begierde in der Schwebe zu halten und damit vermeintlich stabile, wahrnehmungsbildliche Kategorien zu unterlaufen - sie einem Entzug auszusetzen, um beim Betrachter Begehren gegenüber der zeigenden Dimension der Bilder anzuregen.

Das Meer als besonderer Erfahrungsraum eröffnet auf diese Weise den Film. Bereits die Credits entstehen als getippteWorte vor dem Hintergrund der Unterwasserwelt - durch den Akt des Tippens scheint es, als ob die Namen der Schauspieler in diesen Raum hineingeschrieben werden. Dabei schwimmt die Kamera langsam über den sandigen, von den Wellen verwehten Untergrund; sie schweift über wogendes Meeresgras. Der Film gibt sich von Beginn an als bewegter Raum, in dem man suchend eine unbekannte Welt erkundet. Als Zuschauer versucht man, sich zu orientieren, was aber nicht gelingen kann: Die Übergänge sind fließend, die Bewegung schwerelos und ohne Fixpunkte. Dieses Universum ist kaum begrifflich fassbar - und es ist vor allem sinnlich mit einer äußerst haptischen Konnotation versehen.

Das Kapitel „Lucía“ ist allerdings zunächst in der dunklen Stadt und in Leid gefangen. ${ }^{13}$ Erst auf der Insel schiebt sich das glitzernde Meer ins Bild. Als Lucía (Paz Vega) in ein Loch im Felsen fällt und sie durch die besondere, runde Rahmung des Felsens (einem natürlichen Kameraobjektiv ähnlich) auf das azurblaue Meer blickt, kommt „el sexo“ ins Spiel - und mit ihm die Irritation der Zeitebenen; dies, obwohl sich die folgende Liebesszene zwischen Lorenzo (Tristán Ulloa), dem späteren Geliebten Lucías, und Elena (Najwa Nimri) als einfacher Rückblick tarnt.

Man sieht Lorenzo in einem Unterwasserraum schweben. Balken aus Licht und Schatten streifen seinen fast weißen Körper in der nächtlichen Unterwasserwelt von unten nach oben. Im Gegenschuss hinauf sieht man in ein helles Gegenlicht, das seine Strahlen über das Bild, wie einen Trichter drehend, ausbreitet. Dort hinein, vor diese fluide Sonnenerscheinung, schwimmt langsam eine zarte Schattenkontur. Die Bewegung des gleitenden Körpers aus der Untersicht wird auf der Tonebene überlagert von dem genussvollen, sanften Atmen einer Frau. In einer schnelleren Abfolge sieht 


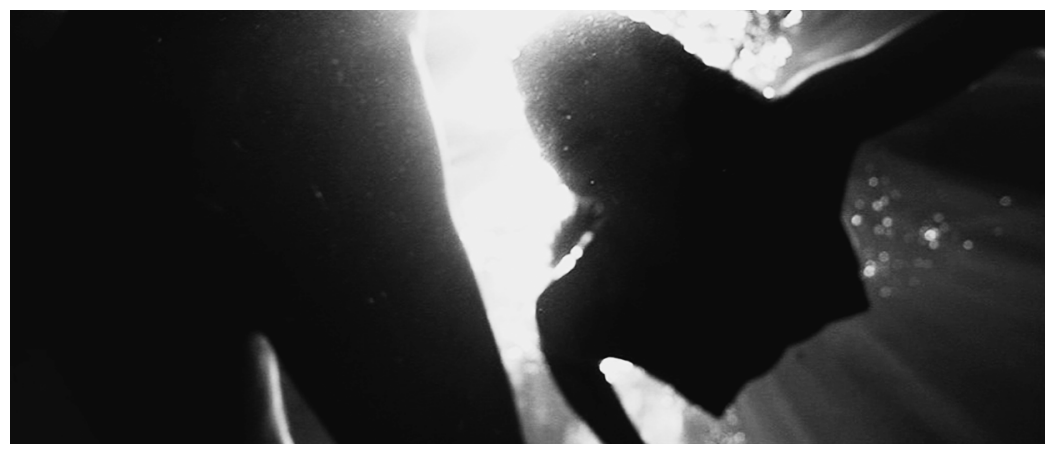

Lucía y el sexo (Lucía und der Sex, F/E 2001)

man die beiden Körperschatten in unterschiedlichen Positionen und Einstellungen miteinander im Gegenlicht verschmelzen - zwischen Schatten und Blendung. Durch den Raum des Wassers und die gleitenden Bewegungen der Körper scheinen die eigentlich fragmentierenden Einstellungen zu einer omnipräsenten, auf die Körper bezogenen Bewegung zu verschmelzen. In dieser Verschmelzung scheinen sie aber auch unfassbar.

Die konkrete Raumerfahrung des Zuschauers, in die „el sexo“ sinnlich transponiert wird, funktioniert auf mehreren Ebenen. Zunächst wird mit der Einstellung auf den frei schwebenden Lorenzo das Gefühl von Schwerelosigkeit etabliert. Auf diese Weise entsteht das Gefühl des „EingetauchtSeins“: Als Zuschauer ist man ganz und gar von der umgebenden Umwelt erfasst. Lorenzos Körper wird über die Lichtstrahlen als Projektionsfläche für die Wahrnehmung des Zuschauers gleitend in den Blick genommen, von den wogenden Bewegungen nachhaltig imprägniert. Diese ondulierenden Momente der Sichtbarkeit übersetzen sich in die Montage der Bewegungen der menschlichen Körper.

Neben dem gleitenden Fluss der Montage und der partiellen Bildausschnitte von den Körpern wird auch noch auf der Licht- und Schattenebene mit Sichtbarkeiten gespielt. Lust und sexuelle Erregung übersetzen sich in ephemere Bewegungen, die wiederum in den Luftblasen als Lichtreflexe vermittelt werden und damit für den Zuschauer das Bild um visuelle Reize anreichern. Sowohl Gegenlicht wie auch das Dunkel sind Grenzerfahrungen der filmischen Wahrnehmbarkeit, in denen das Imaginäre beim Zuschauer aktiviert wird. In der Erfahrung der Blendung durch Gegenlicht wird das Moment des „Überschusses“, des nicht mehr auszuhaltenden „Zu viel“, des nicht mehr zu fassenden Exzesses sinnlich für den Zuschauer spürbar. 
Der so eingeführte Sex und seine auf den Zuschauer gerichtete Verräumlichung im Meer wird zum erzählerischen wie formalen générateur des Films. ${ }^{14}$ Générateur, in Anlehnung an die Begrifflichkeit von Alain RobbeGrillet, heißt, dass wiederkehrende (sinnliche) Momente, „Keimzellen“ gleich, in unterschiedlichsten Variationen die Struktur und Motivik einer Erzählung konzipieren, rhythmisieren und imprägnieren. Der von Medem filmisch vermittelte "el sexo“ ist insofern die zeitlich flottierende filmische „Keimzelle“ für das - zunächst auf der inhaltlichen Ebene -, was am Anfang des Films passiert: In der Sexszene wird die Tochter Luna gezeugt, die dann zur Suche Lorenzos führt und schließlich zu dem tragischen Tod der Tochter, wodurch es zur Trennung von Lorenzo und Lucía kommt. Es folgen der Unfall und der folgenschwere Irrtum Lucías in Bezug auf Lorenzos Tod. An dieser Stelle setzt der Film tatsächlich mit seinem Anfang ein.

In dem räumlichen Prinzip „Meer“ wird Sehnsucht und sexuelle Lust über die Wahrnehmungsstrukturen vermittelt. Dies geschieht über die Auflösung der wahrnehmungsbildlichen Grenzen von Fiktion und Realität ideell ähnlich dem gleitenden Spiel mit den Sichtbarkeiten im Meer. Der Fortgang der filmischen Erzählung ist eng mit Lorenzos schöpferischem und kreativem Akt des Schreibens verquickt. Dies manifestiert sich wahrnehmungsbildlich, wenn sich in Lorenzos entstehender Fiktion "tatsächliche" Ereignisse und Imaginationen auf der ansichtigen Bildebene mischen. So wird die Suche nach Luna vermischt mit den freizügigen Erzählungen der Babysitterin Bélen, was Lorenzos sexuelle Fantasie anregt, in welche wiederum Bilder von Lucía eingebunden werden. So verliert man als Zuschauer den Überblick über die verschiedenen, verschwimmenden Ebenen, wo durchaus die Figuren ihre Identität wechseln können. Das Wahrnehmungsbild fängt in den gleitenden Übergängen an zu flottieren, weshalb Begehren nicht mehr an eine Person oder ein Objekt gebunden ist, sondern offen als "frei bewegt" vermittelt wird. Begehren wird als Grenzen diffundierendes Prinzip erzählt, wodurch die Imagination des Zuschauers stimuliert wird und in das flottierende Spiel der sexuellen, lustvollen Blicke mit hineingezogen wird: Das, was man sieht, ist niemals verlässlich. Es könnte alles auch ganz anders sein (dies legt auch das Ende des Films nahe).

Der Raum des Meeres taucht als variabler, sinnlicher générateur in dieser verworrenen filmischen Erzählung, in der nichts stabilen Kategorien zu unterliegen scheint, in kaum zu erfassender Vielzahl auf: Als tatsächliches Meer, das die Insel umgibt; in symbolischer Form eines Kalenderbildes; als sprachliche Beschreibung; als auditives Zitat oder ganz zeichenhaft in der Zimmerdekoration von Luna. 


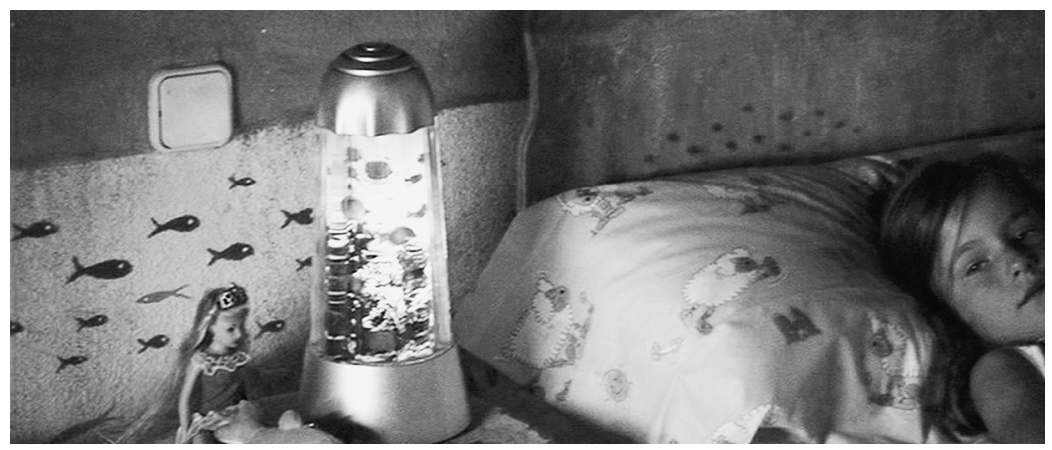

Silvia Llanos in Lucía y el sexo (Lucía und der Sex, F/E 2001)

So unberechenbar und unfassbar das Meer als Raum ist, so sehr gilt dies für seine Bewegungen als Ausdruck von Emotionen unterschiedlichster Art. Als Elena versteht, dass Lucías Lorenzo der Vater ihres Kindes ist, fangen die Kamera und damit der ganze Raum für den Zuschauer an zu wogen. Elena potenziert dies auf der diegetischen Ebene. Ihr ist ganz komisch, sie wankt aus dem Raum. ${ }^{15}$ Das konkrete und emotionale Wogen der Körper ist filmischer Ausdruck einer sexuell und sinnlich-emotional aufgeladenen Verräumlichung in Bewegung, die den gesamten Film als dynamischen, ondulierenden, d. h. wellenbewegten Raum der Schwebe affiziert. ${ }^{16}$ Dabei gerät die zeigende Dimension der filmischen Bilder ebenfalls in eine schwebende Bewegung, was begehrende, lustvolle Fantasien beim Zuschauer anregen und ihn somit emotional zum „Wogen“ bringen kann.

\section{Das Wogen der Bilder}

Ich habe an den vier Beispielfilmen aufgezeigt, wie sich über die räumlichen wie audiovisuellen Qualitäten des Meeres Erfahrungen des Entzugs bzw. des Verlusts in der filmischen Wahrnehmung sinnlich wirksam ausprägen. Bei L'Avventura war es zunächst der bewusste Entzug von verlässlichen Innenräumen und -ansichten. Dies galt auch für die Figuren, deren Bewegungen und Motivationen nicht mehr von einer psychologischen Charakterisierung getragen werden. Vielmehr wurden herkömmliche Kategorien einer Öffnungsbewegung ins Unbestimmte unterworfen. In der besprochenen Sequenz geschieht dies paradigmatisch über die auditive Bewegung des Meeres. Die filmischen Bilder verneinen in dieser Öffnung semantische Festschreibungen. Das, was sie zeigen, vermittelt immer einen Abbruch an Sicherheit. 
In The Bourne Identity wird dem menschlichen Körper die selbstbestimmte Eigenbewegung entzogen. Stattdessen wird er von dem übermächtigen Meeresraum als Bewegungsuniversum inkorporiert. Daran binden sich filmisch besondere Strukturen von Sichtbarkeiten, die keine orientierende Totalübersicht angesichts der Bewegung zulassen. Der menschliche Körper ist nicht mehr bestimmender Fokus. Vielmehr sind nur noch blitzhafte, momenthafte Ein- und Ansichten möglich. Die Fokussierung auf den reinen Bewegungsdrang - des Meeres wie des Films legt die Erfahrung einer filmischen Dauer nahe.

Dies findet sich explizit in John Hustons Moby Dick: Über die Konstruktion von Sichtbarkeitsstrukturen im Bewegungschaos des Meeres, das sich in die Montage übersetzt, wird eine Verlusterfahrung des Bildlichen dezidiert in eine filmische Dauer transponiert.

Lucía y el sexo greift all diese Elemente auf. Der Film profiliert einen Entzug der narrativen Stabilität in der gesamten Raum-Zeitstruktur auf seiner Makroebene. Auf der Mikroebene bedeutet dies vor allem die Auflösung wahrnehmungsbildlicher Sicherheiten. Auf diese Weise wird die Sinnlichkeit filmischer Bilder als Funktionsprinzip im Dienste des lustvollen Sujets ausgestellt und offen funktionalisiert. So lässt sich diese große Affinität zwischen filmischen Bildern und der Bewegung des Meeres ausmachen: Sie wirken über die Gleichzeitigkeit eines Anspruchs des Zuschauers und dem Abbruch, den einem das Bild tut. In dem ständigen, nicht fassbaren Wogen von Bild und Zuschauer entstehen die sinnlichen Empfindungen.

${ }^{1}$ Heinrich von Kleist, „Empfindungen vor Friedrichs Seelandschaft“ (1810), in: Heinrich von Kleist, Erzählungen, Anekdoten, Gedichte, Schriften, hrsg. von Klaus Müller-Salget, Frankfurt/M. 1990, Bd.3, S. 543 f., hier S. 543. (= H. v. K., Sämtliche Werke und Briefe in 4 Bd., hrsg. von Ilse-Marie Barth u. a.). Ursprünglich erschienen in den Abendblättern, 1. 10.1810.

2 So sieht Bernhard Blume Wasser als poetischen Gegenstand mit einem besonderen Potenzial der Vielgestaltigkeit: „Quelle und Bach; Fluß, Strom und Meer; Teich und See; Brunnen und Fontäne; Katarakt und Strudel - eine Fülle von Erscheinungsformen bietet sich dem Gestaltungswillen des Dichters." Bernhard Blume, „Lebendiger Quell und Flut des Todes. Ein Beitrag zu einer Literaturgeschichte des Wassers“ (1966), in: Existenz und Dichtung, hrsg. von Egon Schwarz, Frankfurt/M. 1980, S. 149-166, hier S. 151.

3 Vgl. ebenda, S. 154.

${ }^{4}$ Vgl. ebenda, S. 162.

5 Vgl. ebenda sowie Hartmut Böhme, „Umriß einer Kulturgeschichte des Wassers. Eine Einleitung“, in: Kulturgeschichte des Wassers, hrsg. von Hartmut Böhme, Frankfurt/M. 1988, S. 7-42, hier S. 25. 
${ }^{6}$ Vgl. Bernhard Blume, „Die Kahnfahrt. Ein Beitrag zur Motivgeschichte des 18. Jahrhunderts“ (1957), in: Existenz und Dichtung, hrsg. von Egon Schwarz, Frankfurt/M. 1980, S. 195-236, hier S. $221 \mathrm{f}$.

7 Unter „Bild“ im Kontext des Filmischen verstehe ich hier auch den Fluss der Montage.

8 Blume, „Lebendiger Quell“, (s. Anm. 2), S. $151 \mathrm{f}$.

${ }^{9}$ Vgl. ebenda, S. 152.

10 Eine solche Rückenfigur, die auf das Meer starrt, kommt relativ häufig in Filmen vor. Eva J. M. Schmid schreibt über die Rückenfigur, die sie auch in den Filmen von Tarkowskij ausmacht: „Die Rückenfigur im Bild gibt es schon im 15. Jahrhundert. Aber diese Figur als Projektion des Zuschauers ins Bild zu begreifen, ist erst seit der Romantik möglich. Wir werden aufgefordert, mit den Augen des Dargestellten ins Bild, in die Bildtiefe hinein zu schauen." (Vgl. hierzu v. a. das Werk von Caspar David Friedrich, zum Beispiel Der Mönch am Meer (1808-10) oder Wanderer über dem Nebelmeer (1810)). Eva J. M. Schmid, „Erinnerungen und Frage“, in: Andrej Tarkowskij, hrsg. von PeterW. Jansen und Wolfram Schütte, MünchenWien 1987, S. 43-80, hier S. 62f. Bernhard Kock zitiert Ned Rifkin und thematisiert auf diese Weise die Verbindung von C. D. Friedrich und Antonioni; dies insbesondere im Horizont des Fenster-Motivs. Allerdings stellt Kock Rifkins Befunde infrage. Vgl. Bernhard Kock, Michelangelo Antonionis Bilderwelt. Eine phänomenologische Studie, München 1994, S. 33.

11 Dies ist laut Roger Tailleur eine der zwei entscheidenden Änderungen, die Huston am Ende des Films gegenüber der Romanvorlage vorgenommen hat. Vgl. hierzu Roger Tailleur, „Les chasses de John Huston“ (1957), in: John Huston, hrsg. von Gilles Ciment, Paris 1988, S. 95-103, hier S. 102. Tailleur sieht hier Starbuck in der Tradition der Huston'schen Helden, der sich einer gewissen „honneur tout professionel" verpflichtet sieht.

12 Vgl. hierzu die Ausführungen zur Rolle des Meeres als „Smooth Space“ im Roman Moby Dick in Tamsin Lorraine, „Ahab and Becoming-Whale: The Nomadic Subject in Smooth Space“, in: Deleuze and Space, hrsg. von Ian Buchanan und Gregg Lambert, Edinburgh 2005, S. 159-175.

13 Vgl. Julia Bochnig, „Von der großen Sehnsucht zu fliehen.“ Die Filme von Julio Medem, Remscheid 2005, S. 75.

14 Vgl. Gerda Zeltner, Im Augenblick der Gegenwart. Moderne Formen des französischen Romans, Frankfurt/M. 1974, S. 43-73.

15 Ähnlich verräumlicht wird auch die Freude Lucías, als sie Lorenzo lebendig wiedersieht.

16 Vgl. hierzu Hartmut Böhme: Die Leib-Phänomenologie lege die Frage nahe, ob nicht vielen leiblichen Gefühlen auch eine „Wasser-Natur“, eine „flüssige“ Dynamik eigen sei - wie etwa beim Wogen des Leibes vor dem Aufbrausen des Zorns oder der Lust. Vor dem Hintergrund einer leibphänomenologischen Perspektive sieht Böhme eine tatsächliche „Realanalogie“ gegeben: „Die leiblichen Sensationen etwa in der Liebe sind wasserhaft (...), weil die Struktur leiblicher Liebe und die Struktur der leiblichen Wassererfahrung (...) identisch sind.“ Böhme, „Umriß einer Kulturgeschichte des Wassers“ (s. Anm. 5), S. 24. 